\title{
FARMACOGENÉTICA CARDIOVASCULAR
}

\author{
CARDIOVASCULAR PHARMACOGENETICS
}

Valéria C. Sandrim¹, Vânia B. Rezende ${ }^{2}$, José Eduardo Tanus-Santos ${ }^{3}$

\begin{abstract}
1Pós-doutoranda em Farmacologia. Faculdade de Medicina de Ribeirão Preto - USP. ${ }^{2}$ Pós-graduanda em Farmacologia. Faculdade de Ciências Médicas - UNICAMP. ${ }^{3}$ Docente. Departamento de Farmacologia. Faculdade de Medicina de Ribeirão Preto - USP.

CorresPondência: José Eduardo Tanus dos Santos. Departamento de Farmacologia, Faculdade de Medicina de Ribeirão Preto, USP. Av. Bandeirantes, 3900 - CEP: 14049-900 / Ribeirão Preto, SP, Brazil. FAX: +55 1636332301 / Phone: +55 1636023163 / E-mail: tanus@fmrp.usp.br
\end{abstract}

Sandrim VC, Rezende VB, Tanus-Santos JE. Farmacogenética cardiovascular. Medicina (Ribeirão Preto) 2006; 39 (4): 535-42.

RESUMO: A variabilidade de resposta à droga entre pacientes é parcialmente devida à composição genética única que cada indivíduos apresenta. O ramo da Farmacologia Clínica que estuda tais variabilidades é chamado de farmacogenética. Variações genéticas (chamadas de polimorfismos) podem modificar tanto parâmetros farmacocinéticos (por alterarem a atividade de enzimas importantes no metabolismo de fármacos) quanto parâmetros farmacodinâmicos (por modificar a afinidade de um receptor pelos agonistas ou antagonistas). Atualmente, a farmacogenética vem sendo valorizada como uma ferramenta útil na busca de terapêuticas melhores. Esta revisão enfoca a farmacogenética de drogas de ação cardiovascular. Especificamente, alguns casos clínicos ilustrativos de doenças cardiovasculares serão apresentados e comentados quanto às suas possíveis bases farmacogenéticas.

Descritores: Farmacogenética. Farmacogenômica. Polimorfismo Genético. Doenças Cardiovasculares.

\section{INTRODUÇÃO}

A partir do século passado foi observado um aumento na expectativa de vida da população de um modo geral. Entretanto, este aumento foi acompanhado de alterações no estilo de vida e nos hábitos alimentares, culminando numa elevação da incidência de doenças cardiovasculares na população e, conseqüentemente, no aumento da necessidade de desenvolvimento de novas terapias farmacológicas. Contudo, as respostas a estes tratamentos exibem diferenças entre os pacientes, sendo que, enquanto em alguns indivíduos apresentam respostas efetivas, outros apresentam ausência de resposta ou mesmo toxicidade inesperada. Neste sentido, a busca de ferramentas terapêuticas mais eficazes e seguras que pudessem predizer quais pacientes responderão ou não a uma terapia é extremamente válida.

Algumas condições como doenças concomitantes (especialmente as renais e hepáticas), interações medicamentosas, idade, gravidez, tabagismo, além de fatores ambientais, podem influenciar a resposta a uma droga. Atualmente, aliada aos avanços da genética e o sequenciamento do genoma humano, tem sido muito destacada a influência de variações genéticas nas respostas às drogas. $\mathrm{O}$ estudo deste ramo da farmacologia é chamado de farmacogenética. Acreditase que a farmacogenética poderá auxiliar na identificação de quais pacientes se beneficiarão com uma dada terapia com um menor risco de efeitos adversos, 
ou seja, possibilitará uma terapia individualizada mais segura.

De maneira geral, uma droga sofre modificações para que possa atingir o local de ação, antes de ser excretada. Estão envolvidas neste processo várias enzimas, as quais são proteínas que são codificadas por genes compostos por DNA. Este, por sua vez, apresenta variações genéticas interindividuais.

As variações genéticas são chamadas de polimorfismos quando presentes numa frequiência maior que $1 \%$ na população. Elas podem ser de vários tipos (deleção/inserção, SNPs, VNTR, entre outros). Entretanto, o tipo mais comum, responsável por aproximadamente $90 \%$ da variabilidade entre os indivíduos, é o SNP (single nucleotide polymorphism). Um SNP acontece quando, por exemplo, verifica-se uma substituição de uma base "A"(adenina) por "T"(timidina) num determinado nucleotídeo do genoma.

Na verdade, milhões de polimorfismos vêm sendo identificados e catalogados recentemente. No entanto, apenas alguns deles apresentam conseqüências funcionais, ou seja, acarretam alterações na expressão gênica, na síntese e atividade biológica das proteínas que codificam, podendo alterar as respostas às drogas. Por exemplo: quando um SNP ocorre numa região codificadora do gene de um receptor, a substituição de uma base do DNA por outra pode levar a uma alteração da estrutura do receptor e alterar a interação deste receptor ao seu ligante endógeno e/ou aos fármacos. Já SNPs localizados na região promotora do gene podem alterar a expressão gênica, alterando a quantidade de proteínas envolvidas nas respostas às drogas ${ }^{1}$. Por exemplo, existe um SNP clinicamente relevante que ocorre na região promotora do gene da sintetase endotelial do óxido nítrico (eNOS), o qual tem sido associado com risco aumentado de doença coronariana ${ }^{2}$. Este SNP é descrito como T- ${ }^{786} \mathrm{C}$, o que indica que a base de número -786 da região promotora do gene da eNOS pode conter uma base $\mathrm{T}$ (alelo mais comum) ou C (alelo mais raro). Foi demonstrado que o variante $\mathrm{C}$ acarreta $50 \%$ de redução na eficiência do promotor comparado ao variante $\mathrm{T}^{3}$. Desta maneira, estas variações genéticas podem alterar funções importantes em várias vias biológicas acarretando maior ou menor susceptibilidade no desenvolvimento de doenças, além de modularem mecanismo de ação de drogas.

Nesta revisão, pretendemos descrever alguns casos clínicos que evidenciam como algumas variações genéticas podem modular a terapia cardiovascular.

\section{CASOS CLÍNICOS}

Caso 1: Terapia com varfarina é iniciada em um paciente com trombose venosa. Uma semana depois, o paciente passa a apresentar hemorragia intracerebral e INR (international normalized ratio) de 11 (normal 0,2-1,8, estabelecido pela OMS e o Comitê Internacional sobre Trombose e Hemostasia).

Caso 2: Um homem com 52 anos de idade e histórico familiar de doenças cardiovasculares foi ao consultório médico para exames de rotina. Para sua surpresa, o colesterol apresentou níveis séricos de 9.0mmol/L, sendo que os desejáveis são valores menores que 5.0mmol/L. Seu médico determinou que fosse medicado com pravastatina, um medicamento da classe das estatinas. Após alguns meses de tratamento e dieta balanceada, o paciente ficou extremamente satisfeito com os níveis de seu colesterol que baixaram para $5.5 \mathrm{mmol} / \mathrm{L}$.

Caso 3: Um paciente é tratado há anos com quatro anti-hipertensivos, incluindo um diurético. Entretanto, seus níveis da pressão sanguínea não atingem valores ideais, permanecendo em 170/120 mmHg. Possíveis causas de resistência terapêutica foram avaliadas e excluídas, caracterizando o quadro clínico como hipertensão resistente à terapia (refratária).

Caso 4: Terapia com sotalol é iniciada em um homem com 78 anos de idade que apresenta fibrilação atrial. Dois dias depois, após um episódio de sincope, o paciente passou a apresentar ritmo sinusal com um intervalo $Q T$ de 0,7 segundo (intervalo QT normal: 0,42 segundo). Torsades de pointes foram observadas no ECG.

\section{DISCUSSÃO DOS CASOS}

\section{Caso 1}

O sistema de enzimas do citocromo P450 é o principal grupo de enzimas envolvidas no metabolismo de drogas. Tal importância justifica a enorme quantidade de estudos farmacogenéticos enfocando várias de suas enzimas ${ }^{4}$. Os polimorfismos presentes nos genes que codificam estas enzimas afetam suas atividades enzimáticas. Por exemplo: dois polimorfismos comuns da CYP2C9 (alelo *2 e*3) causam reduções 
da atividade enzimática de $30 \%$ e $80 \%$, respectivamente. Desta maneira, os indivíduos de uma população podem ser caracterizados, segundo o variante genético que apresentam, como metabolizadores normais, metabolizadores lentos e metabolizadores rápidos, conforme a capacidade de metabolização de drogas. Esta variabilidade entre os indivíduos traz conseqüências diretas sobre os parâmetros farmacocinéticos.

Uma vez que os esquemas de dosagens de drogas são obtidos a partir de estudos clínicos de fase I e II, os quais são realizadas com um número pequeno de indivíduos, é possível que quando utilizado em grande escala no mercado, este medicamento cause efeitos variáveis não previstos, pois poderá haver indivíduos portadores de polimorfismos que afetem enzimas importantes para o metabolismo da droga. Assim, tais indivíduos requereriam adequação de dose. Caso contrário, poderia haver toxicidade ou ineficácia desta droga em indivíduos portadores de tais variantes.

A Tabela I mostra algumas das principais drogas de ação cardiovascular metabolizadas pelas enzimas do sistema citocromo P450. As enzimas CYP3A4 e CYP2D6 são as mais importantes no metabolismo das drogas utilizadas no tratamento de doenças cardiovasculares. Podemos notar que vários bloqueadores de canais de cálcio, estatinas e antagonistas dos receptores tipo 1 da angiotensina II são metabolizados por estas enzimas ${ }^{5}$. Assim, polimorfismos que alterem a atividade enzimática destas enzimas modificam significativamente o perfil farmacocinético destas drogas, potencialmente afetando a resposta terapêutica.

Um bom exemplo de como polimorfismos genéticos presentes em enzimas metabolizadores de drogas podem ter alto impacto clínico é o caso clínico 1 apresentado acima. A Varfarina é um anticoagulante amplamente usado na terapia de complicações trombovenosas e após cirurgias cardíacas. Esta droga é um composto com atividade anticoagulante e é rapi- damente removida da circulação pela CYP2C $9^{6}$. Um número de variantes alélicos neste gene é conhecido, sendo os mais comuns: CYP2C9*2 (o número que se segue ao sinal "* " indica qual variante genética da CYP2C9 estamos tratando) caracterizado pela substituição de uma cisteína por uma arginina no aminoácido 144 desta enzima. Esta troca provoca diminuição em $88 \%$ atividade enzimática. Um outro variante, o CYP2C9*3, onde uma leucina é substituída pela isoleucina no resíduo 359 , exibe apenas $5 \%$ da eficiência do variante mais comum - leucina ${ }^{6}$. Sendo assim, portadores dessas variantes (arginina e isoleucina) metabolizam a varfarina mais lentamente, levando a um aumento dos níveis plasmáticos do anticoagulante varfarina, o que aumenta o risco de sangramentos, como observado no indivíduo do caso 1 . Por isso, os pacientes com uso desse medicamento devem fazer monitoramento freqüente dos níveis de varfarina plasmática.

De maneira semelhante à varfarina, foi demonstrado que polimorfismos genéticos da CYP2C9 afetam a resposta ao irbesartan (antagonista dos receptores tipo 1 da angiotensina II), sendo que metabolizadores lentos para a CYP2C9 apresentaram redução em $14 \%$ dos níveis da pressão arterial diastólica, enquanto que os metabolizadores normais têm redução de apenas $7 \%{ }^{7}$.

Outro exemplo importante se refere ao uso de beta-bloqueadores. Tais drogas são metabolizadas, na sua maioria, pela enzima CYP2D6. Um estudo mostrou que a ocorrência de polimorfismo no gene que codifica esta enzima reduziu em $60 \%$ a taxa de depuração plasmática de carvedilol, o que resultou em um aumento da sua biodisponibilidade de mais de $150 \%$. Ainda, outro estudo mostrou que indivíduos metabolizadores lentos para a enzima CYP2D6 têm 5 vezes mais chances de apresentar efeitos colaterais associados ao uso de metoprolol quando comparados a metabolizadores normais ${ }^{8,9}$.

Tabela I: Drogas utilizadas no tratamento de problemas cardiovasculares que são metabolizadas por enzimas do citocromo P450

\begin{tabular}{cl}
\hline Citocromo P450 & Drogas metabolizadas \\
\hline CYP3A4 & $\begin{array}{l}\text { Amlodipina, atorvastatina, cerivastatina, diltiazem, felodipina, lercanidipina, lidocaina, } \\
\text { losartan, lovastatina, nicarnidipina, nifedipina, nimodipina, nisoldipina, nitrendipina, } \\
\text { quinidina, simvastatina, verapamil }\end{array}$ \\
& $\begin{array}{l}\text { Carvedilol, debrisoquina, encainida, flecainida, lidocaina, metoprolol, mexiletina, } \\
\text { propafenona, propranolol, timolol }\end{array}$ \\
CYP2C9 & Fluvastatina, irbesartan, losartan, torsemida, varfarina \\
\hline
\end{tabular}


O conhecimento da existência desses polimorfismos de relevância clínica, como no caso do metabolismo da varfarina, nos leva a pensar na importância da farmacogenética e/ou farmacogenômica, a qual poderá auxiliar na prática clínica ao evitar tais problemas com simples testes de genotipagens (determinação de quais marcadores genéticos específicos o paciente tem).

\section{Caso 2}

A principal reação na biossíntese do colesterol é catalisada pela enzima microssomal 3-hidroximetilglutaril coenzima A (HMG-CoA) redutase. Esta enzima condiciona o passo limitante na síntese do colesterol ${ }^{10}$. As estatinas, uma das drogas com maior mercado mundial, são inibidores competitivos da HMGCoA redutase. Seus principais efeitos incluem principalmente diminuição dos níveis plasmáticos do colesterol de baixa densidade (LDL, vulgarmente conhecido como "colesterol ruim") ${ }^{11}$. O caso 2 citou um paciente que respondeu de forma positiva ao tratamento. Entretanto, há uma considerável variação interindividual na resposta a esses medicamentos (Figura 1). Estimase que fatores genéticos possam ser responsáveis por cerca de 20 a $95 \%$ dessa variabilidade.

Vários estudos avaliaram diferentes respostas terapêuticas com o uso de inibidores da HMG-Coa redutase $^{12,13,14}$. Entretanto, a maioria destes estudos enfocou principalmente o efeito de polimorfismos sobre o metabolismo (CYP450), tanto do colesterol quanto das estatinas ${ }^{15}$.

Em humanos, pelo menos metade de todas as drogas são metabolizadas por enzimas da família da

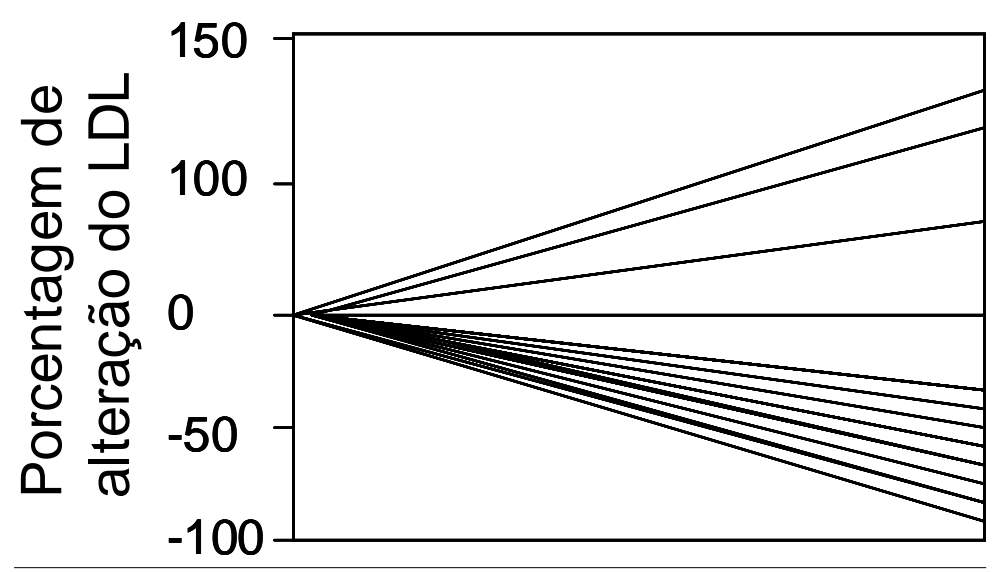

Figura 1 - Porcentagem de alteração (redução ou aumento) dos níveis de triglicérideos (TG) individuais em resposta ao tratamento com estatina. Estes dados não foram retirados de nenhum estudo específico, apesar de vários estudos clínicos relatam diferentes respostas às estatinas.
CYP3A, que apresenta duas isoformas: CYP3A4, sendo a principal forma hepática, e a CYP3A $5^{16}$. Existe um polimorfismo na região promotora (A-290G) do gene da CYP3A4 que afeta a regulação da transcrição gênica da enzima CYP3A4 e, conseqüentemente, o metabolismo de drogas como algumas estatinas (lovastatina, sinvastatina e atorvastatina) ${ }^{15}$. Neste sentido, um estudo clínico mostrou que este polimorfismo afeta a resposta à atorvastatina, observando-se diferentes níveis de LDL plasmático após tratamento, conforme o genótipo específico ${ }^{17}$.

\section{Caso 3}

O tratamento da hipertensão arterial é geralmente baseado em "tentativa e erro", onde a escolha do anti-hipertensivo é geralmente fundamentada na experiência pessoal do médico e em resultados obtidos a partir de estudos clínicos. Tais estudos levam a conclusões relativas a população em geral, mas nem sempre são aplicáveis a perfis individuais. Agravando o quadro, a resposta aos anti-hipertensivos é altamente variável e, às vezes, ausente, como no caso 3. Desta maneira, estabelecer marcadores genéticos de resposta a anti-hipertensivos seria extremamente interessante, pois uma terapia mais personalizada seria prescrita para cada paciente individualmente, além de contribuir para o desenvolvimento de novos fármacos com diferentes mecanismos de ação.

A seguir serão apresentados alguns exemplos de estudos que avaliam a influência de variantes genéticos sobre a resposta a anti-hipertensivos (Tabela II), divididos segundo suas classes ${ }^{18,19}$. Optamos por abordar apenas polimorfismos que aparentemente acarretam modificações funcionais, por exemplo, alterando a síntese de segundos mensageiros intracelulares. Tais estudos podem fortalecer os resultados obtidos na clínica.

- Diuréticos: os diuréticos tiazídicos são considerados drogas de primeira escolha para a maioria dos hipertensos. Entretanto, a resposta aos diuréticos pode variar. Como as ações farmacológicas dos diuréticos são amplas, e vários polimorfismos podem influenciar suas respostas. Até o momento, dentre todos os polimorfismos avaliados, o mais promissor parece ser um SNP localizado no gene que codifica a proteína aducina que apresenta papel importante na regulação da reabsorção de sódio nos túbu- 


\begin{tabular}{|c|c|c|c|c|}
\hline Gene & Lócus & Alteração & Droga & Resultados \\
\hline \multirow[t]{2}{*}{$\alpha$-aducina } & $\mathrm{G}^{614} \mathrm{~T}$ & Gly ${ }^{460}$ Trp & $\mathrm{HCTZ}$ & $\begin{array}{l}\text { Gly/Gly - Gly/Trp; } \triangle \mathrm{MPA}:-15,-7 \\
(P=0,002)\end{array}$ \\
\hline & & & $\mathrm{HCTZ}$ & $\begin{array}{l}\text { Gly/Gly - Gly/Trp; } \triangle \text { MPA: -6; } 12 \\
(P=0,003)\end{array}$ \\
\hline \multirow[t]{2}{*}{ GNB3 } & $\mathrm{C}^{825 T}$ & & HCTZ & $\begin{array}{l}\mathrm{C} / \mathrm{C}-\mathrm{C} / \mathrm{T}-\mathrm{T} / \mathrm{T} ; \Delta \mathrm{PSS}:-10,-14,-16 \\
(\mathrm{P}<0,001) ; \Delta \mathrm{PSD}:-6,-8,-10 \\
(\mathrm{P}<0,001)\end{array}$ \\
\hline & & & Atenolol & $\begin{array}{l}\mathrm{C} / \mathrm{C}-\mathrm{C} / \mathrm{T}+\mathrm{T} / \mathrm{T} ; \Delta \mathrm{PSS}:-25,-17 \\
(\mathrm{P}=0,003) ; \Delta \mathrm{PSD}:-19,-16 \\
(\mathrm{P}=0,027), \Delta \mathrm{PAM}:-21,-16 \\
(\mathrm{P}=0,008)\end{array}$ \\
\hline \multirow[t]{4}{*}{ ECA } & Íntron 16 & Inserção (I)/Deleção (D) & $\begin{array}{c}\text { HCTZ } \\
\text { Lisinopril }\end{array}$ & $\begin{array}{l}\mathrm{I} / \mathrm{I}-\mathrm{I} / \mathrm{D} ; \Delta \mathrm{MPA}:-10,-6(\mathrm{P}=0,02) \\
\mathrm{NS}\end{array}$ \\
\hline & & & Imidapril & $\begin{array}{l}\text { I/I - I/D+D/D DPSS: NS } \\
\Delta P S D:-12,-8(P=0,067)\end{array}$ \\
\hline & & & Perindopril & NS \\
\hline & & & Irbesartan & $\begin{array}{l}\mathrm{I} / \mathrm{I}-\mathrm{l} / \mathrm{D}+\mathrm{D} / \mathrm{D} \Delta \mathrm{PSS}:-24,-12 \\
(\mathrm{P}=0,087) \Delta \mathrm{PSD}:-18,-7 \quad(\mathrm{P}=0,009)\end{array}$ \\
\hline \multirow[t]{3}{*}{ AGT } & $\mathrm{T}^{1198} \mathrm{C}$ & Met $^{235} \mathrm{Thr}$ & Atenolol & 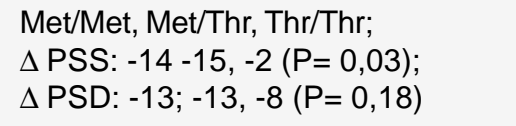 \\
\hline & & & Atenolol & NS \\
\hline & & & Irbesartan & $\begin{array}{l}\text { Met/Met - Met/Thr - Thr/Thr; } \\
\text { DPSS: -12; -20; -8 }(P=0,12) ; \\
\text { DPSD: -6; -12; -4 }(P=0,04)\end{array}$ \\
\hline \multirow[t]{2}{*}{$\beta 1 \mathrm{AR}$} & $\mathrm{G}^{1165} \mathrm{C}$ & Gly ${ }^{389}$ Arg & Metoprolol & $\begin{array}{l}\text { Arg/Arg - Gly/Arg; } \triangle P S D:-12,-5 \\
(P=0,012)\end{array}$ \\
\hline & & & Atenolol & NS \\
\hline
\end{tabular}

los renais. A presença deste SNP causa alteração do aminoácido glicina por triptofano no aminoácido 460 da proteína (Gly460Trp). Uma vez que portadores do variante Trp aparentemente apresentam maior reabsorção tubular de sódio, espera-se que estes indivíduos respondam melhor ao tratamento com HCTZ (que diminui a absorção renal de sal) quando comparados com os indivíduos Gly/Gly ${ }^{20}$. De fato, esta hipótese foi confirmada em vários estudos que demonstram, em média, redução da pressão arterial média mais intensa após tratamento com HCTZ em portadores do variante Trp comparados aos não portadores ${ }^{19}$.

- $\beta$-bloqueadores: a ação dos $\beta$-bloqueadores se dá pela diminuição do inotropismo e cronotro- pismo cardíaco e pela redução da liberação de renina pelas células justaglomerulares. Devido à variabilidade individual encontrada com o uso destes medicamentos, principalmente relacionada à idade e raça, $\mathrm{o}$ interesse em se encontrar bases genéticas para esta variabilidade é muito grande. Por exemplo, que o polimorfismo Arg389 (Gly389Arg) localizado no gene que codifica este receptor está associado a aumento da atividade da adenilato ciclase e da produção de AMPc após ligação do agonista ${ }^{21}$. Assim, poderíamos sugerir que hipertensos portando este alelo responderiam melhor à terapia com $\beta$-bloqueadores (que competem com o agonista pela ligação ao receptor) quando comparados aos portadores de Gly. Esta sugestão foi con- 
firmada em alguns estudos. Num destes, a administração de metoprolol reduziu diferentemente a pressão diastólica nos indivíduos com o genótipo Arg/Arg $(-12 \mathrm{mmHg})$ e indivíduos portadores do variante Gly (-5 mmHg Gly/Arg) ${ }^{21}$.

- Inibidores da enzima conversora de angiotensina: inibidores da enzima conversora de angiotensina (iECA) compreendem uma classe de antihipertensivos que apresentam excelente eficácia no tratamento da insuficiência cardíaca e hipertensão. No entanto, como observado na prática clínica, a resposta a estes medicamentos é bem variável. O polimorfismo mais estudado no gene da ECA é o I/D (inserção/deleção) encontrado no intron 16. Este polimorfismo é caracterizado pela inserção ou deleção de uma seqüência de 287 nucleotídeos. Foi observado que indivíduos homozigóticos para o alelo D (deleção) apresentam altos níveis da enzima conversora ${ }^{22}$. Assim, seria esperado que estes indivíduos respondessem mais eficientemente aos iECA do que os portadores do variante I (inserção), uma vez que teoricamente eles apresentam maior nível de ECA. No entanto, o efeito desta classe de anti-hipertensivos parece não ser modulado por este polimorfismo genético, apesar de alguns estudos sugerirem maior redução da pressão sanguínea em indivíduos $\mathrm{DD}^{19}$.

- Bloqueadores do receptor 1 da angiotensina: esta classe de anti-hipertensivo oferece um mecanismo alternativo para inibição do sistema reninaangiotensina-aldosterona, por atuarem bloqueando a ação da angiotensina II. Desta maneira, o efeito desta classe de anti-hipertensivos poderia ser modulado por polimorfismos localizados no angiotensinogênio, na ECA e no próprio receptor AT1. Os polimorfismos $\mathrm{Met}^{235} \mathrm{Thr}$ (angiotensinogênio) e I/D (ECA) parecem influenciar na resposta ao Irbesartan, apesar de alguns estudos demonstrarem que isto não acontece ${ }^{19}$.

- Bloqueadores de canais de cálcio: com relação aos bloqueadores dos canais de cálcio, até o momento, poucos estudos avaliaram a influência de variantes genéticos sobre o efeito destes medicamentos ${ }^{19}$.

\section{Caso 4}

Arritmias cardíacas graves (do tipo Torsade de Pointes) podem ocorrer durante tratamento com drogas que prolongam o intervalo QT, tais como a quinidina ou o sotalol. Recentemente, foram identificadas mutações genéticas de genes que codificam canais iônicos importantes para a repolarização das fibras cardía- cas. Este processo de repolarização ventricular é extremamente importante e, temporalmente, bem definido. Entretanto, indivíduos portadores destas mutações apresentam intervalos QT prolongado, aumentando em muito a sua susceptibilidade a arritmias cardíacas, especialmente quando estes indivíduos passam a usar drogas que prolongam ainda mais o intervalo $\mathrm{QT}^{23}$. Atualmente, existem exemplos bem documentados, tais como do caso 4, de indivíduos que apresentam mutações nestes genes e que, até o contato com a droga, não manifestam clinicamente o prolongamento do intervalo QT, o que dificulta a prescrição médica adequada. Portanto, a genotipagem para estas mutações poderia evitar que indivíduos com susceptibilidade genética a intervalos QT prolongados tomassem drogas que prolongassem ainda mais este intervalo, levando a arritmias potencialmente fatais ${ }^{23}$.

\section{Perspectivas Futuras}

A Farmacogenética parece se encaminhar numa direção progressivamente mais próxima da prática clínica. Embora alguns defendam o contrário, estamos nos aproximando dos dias em que teremos um tratamento individualizado baseado em marcadores genéticos individuais para cada paciente $e^{24,25,26}$. As conquistas tecnológicas já estão viabilizando a determinação de vários marcadores genéticos para cada paciente, antes mesmo de se iniciar um tratamento farmacológico. Isto pode ter grande valor porque a informação genética gerada é válida pelo resto da vida de cada paciente, uma vez que os determinantes genéticos não mudam com o tempo.

Vários desafios devem ser superados para o sucesso da abordagem Farmacogenética. Talvez o maior deles diga respeito à validação clínica consistente de marcadores genéticos realmente capazes de prever respostas favoráveis ou toxicidade a drogas ${ }^{24,25,26}$.

É possível que a indústria farmacêutica passe a desenvolver drogas cujo uso seja restrito a indivíduos portadores de determinados marcadores genéticos. Isto poderá ter implicações econômicas e sociais que transcendem os aspectos científicos. Neste sentido, sabemos que vários dos polimorfismos genéticos conhecidos apresentam marcantes diferenças quanto a sua distribuição entre os diferentes grupos raciais ou étnicos. Assim, há várias preocupações quanto a aspectos éticos envolvidos nas decisões de se desenvolverem drogas mais úteis a determinados grupos étnicos de maior poder econômico, em detrimento daqueles mais desprotegidos socialmente ${ }^{24}$. 
Sandrim VC, Rezende VB, Tanus-Santos JE. Cardiovascular Pharmacogenetics. Medicina (Ribeirão Preto) 2006; 39 (4): 535-42.

ABSTRACT: The widely acknowledged variability in drug response is partiality due to differences in genetic background. Pharmacogenetics is a branch within the field of Clinical Pharmacology focused on the study of how genetic differences affect drug responses. Genetic variations (called polymorphisms) may change pharmacokinetic parameters (by altering enzymes involved in drug metabolism), and pharmacodynamic parameters (by altering receptor affinity by agonist and antagonist drugs). Presently, pharmacogenetics has been valued as a potentially useful tool in finding better therapeutics. This review is mostly concerned with cardiovascular pharmacogenetics. Clinical cases will be described and their possible pharmacogenetic implications will be briefly commented on.

Keywords: Pharmacogenetics. Pharmacogenomics. Polymorphism, Genetic. Cardiovascular Diseases.

\section{REFERÊNCIAS}

1 - Mooser V, Waterworth DM, Isenhour T, Middleton L. Cardiovascular pharmacogenetics in the SNP era. J Thromb Haemost 2003; 1(7):1398-402.

2 - Hingorani AD, Liang CF, Fatibene J, Lyon A, Monteith S, Parsons $A$, et al. A common variant of the endothelial nitric oxide synthase (Glu298Asp) is a major risk factor for coronary artery disease in the UK. Circulation 1999;100 (14): 1515-20.

3 - Nakayama M, Yasue H, Yoshimura M, Shimasaki Y, Kugiyama $\mathrm{K}$, Ogawa $\mathrm{H}$, et al. T-786-C mutation in the 5'-flanking region of the endothelial nitric oxide synthase gene is associated with coronary spasm. Circulation 1999;99(22):2864-70.

4 - Ingelman-Sundberg M. Pharmacogenetics of cytochrome P450 and its applications in drug therapy: the past, present and future. Trends Pharmacol Sci 2004;25 (4):193-200.

5 - Schaefer BM, Caracciolo V, Frishman WH, Charney P. Gender, ethnicity and genetics in cardiovascular disease: part 1: Basic principles. Heart Dis 2003;5(2):129-43.

6 - Sanderson S, Emery J, Higgins J. CYP2C9 gene variants, drug dose, and bleeding risk in warfarin-treated patients: a HuGEnet systematic review and meta-analysis. Genet Med 2005;7 (2): 97-104.

7 - Siffert W. Cardiovascular pharmacogenetics: on the way toward individually tailored drug therapy. Kidney Int 2003(Suppl 84):S168-71.

8 - Brookes AJ. The essence of SNPs. Gene 1999;234 (2): 17786.

9 - Anderson JL, Carlquist JF, Horne BD, Muhlestein JB. Cardiovascular pharmacogenomics: current status, future prospects. J Cardiovasc Pharmacol Ther 2003;8(1):71-83.

10 - Endo A. The discovery and development of HMG-CoA reductase inhibitors. J Lipid Res 1992;33(11):1569-82.

11 - Brown MS, Goldstein JL. A receptor-mediated pathway for cholesterol homeostasis. Science 1986;232 (4746):34-47.
12 - Gerdes LU, Gerdes C, Kervinen K, Savolainen M, Klausen IC, Hansen PS, et al. The apolipoprotein epsilon4 allele determines prognosis and the effect on prognosis of simvastatin in survivors of myocardial infarction : a substudy of the Scandinavian simvastatin survival study. Circulation 2000; 101 (12): 1366-71.

13 - Bray PF, Cannon CP, Goldschmidt-Clermont P, Moye LA, Pfeffer MA, Sacks FM, et al. The platelet PI(A2) and angiotensin-converting enzyme (ACE) $D$ allele polymorphisms and the risk of recurrent events after acute myocardial infarction. Am J Cardiol 2001;88 (4):347-52.

14 - de Maat MP, Jukema JW, Ye S, Zwinderman AH, Moghaddam $\mathrm{PH}$, Beekman M, et al. Effect of the stromelysin-1 promoter on efficacy of pravastatin in coronary atherosclerosis and restenosis. Am J Cardiol 1999;83 (6): 852-6.

15 - Bolego C, Baetta R, Bellosta S, Corsini A, Paoletti R. Safety considerations for statins. Curr Opin Lipidol 2002; 13 (6): 637-44.

16 - Siest G, Ferrari L, Accaoui MJ, Batt AM, Visvikis S. Pharmacogenomics of drugs affecting the cardiovascular system. Clin Chem Lab Med 2003;41 (4): 590-9.

17 - Kajinami K, Brousseau ME, Ordovas JM, Schaefer EJ. CYP3A4 genotypes and plasma lipoprotein levels before and after treatment with atorvastatin in primary hypercholesterolemia. Am J Cardiol 2004; 93 (1):104-7.

18 - Kurland L, Lind L, Melhus H. Using genotyping to predict responses to anti-hypertensive treatment. Trends Pharmacol Sci 2005;26(9):443-7.

19 - Koopmans RP, Insel PA, Michel MC. Pharmacogenetics of hypertension treatment: a structured review. Pharmacogenetics 2003;13(12):705-713.

20 - Bianchi G, Ferrari P, Staessen JA. Adducin polymorphism: detection and impact on hypertension and related disorders. Hypertension 2005;45(3):331-40.

21 - Mason DA, Moore JD, Green SA, Liggett SB. A gain-offunction polymorphism in a G-protein coupling domain of the human beta1-adrenergic receptor. J Biol Chem 1999; 274 (18): 12670-4. 
22 - Rigat B, Hubert C, Alhenc-Gelas F, Cambien F, Corvol P, Soubrier F. An insertion/deletion polymorphism in the angiotensin Iconverting enzyme gene accounting for half the variance of serum enzyme levels. J Clin Invest 1990;86(4):1343-6.

23 - Splawski I, Timothy KW, Tateyama M, Clancy CE, Malhotra A, Beggs $\mathrm{AH}$, et al. Variant of SCN5A sodium channel implicated in risk of cardiac arrhythmia. Science 2002; 297 (5585):1333-6.
24 - Tanus-Santos JE. Farmacogenética em Cardiologia. Rev SOCESP 2004; 14(3):530-8.

25 - Sandrim VC, Tanus-Santos JE. Farmacogenômica em hipertensão: aspectos fisiológicos. Hipertensão 2006; 9(1): 4-8.

26 - Sandrim VC, Tanus-Santos JE. Farmacogenômica em hipertensão: aspectos fisiopatológicos. Hipertensão 2006, 9 (1): 9-14. 